\title{
Rings of Idempotent Stable Range One
}

\author{
Zhou Wang • Jianlong Chen • Dinesh Khurana • \\ Tsit-Yuen Lam
}

Received: 31 December 2010 / Accepted: 2 February 2011 / Published online: 22 February 2011

(C) The Author(s) 2011. This article is published with open access at Springerlink.com

\begin{abstract}
We show that in a ring of stable range 1, any (von Neumann) regular element is clean. Our main results also imply that any unit-regular ring has idempotent stable range 1 (and is therefore clean), and that a semilocal ring has idempotent stable range 1 if and only if it is semiperfect.
\end{abstract}

Keywords Idempotent stable range one • Stable range one • Regular elements • Unit regular rings $\cdot$ Clean rings

Mathematics Subject Classifications (2010) $16 \mathrm{D} 70 \cdot 16 \mathrm{E} 50$

\section{Introduction}

A goal of this short note is to prove that, in a ring $R$ of stable range 1, any (von Neumann) regular element $a \in R$ is clean; that is, $a$ can be written as the sum of an

Presented by Kenneth Goodearl.

Z. Wang $(\varangle) \cdot$ J. Chen

Department of Mathematics, Southeast University, Nanjing 210096,

People's Republic of China

e-mail: zhouwang@seu.edu.cn

J. Chen

e-mail: jlchen@seu.edu.cn

D. Khurana

Faculty of Mathematics, Indian Inst. of Sci. Edu. \& Res., Mohali, MGSIPA Transit Campus, Sector 26, Chandigarh 160 019, India

e-mail: dkhurana@iisermohali.ac.in

T.-Y. Lam

Department of Mathematics, University of California, Berkeley, CA 94720, USA

e-mail: lam@math.berkeley.edu 
idempotent and a unit in $R$. The main results of the paper, given in Theorem 3.1 and Theorem 3.3, are actually more precise than this, and can be described in more detail after recalling a few key definitions.

An element $a$ in any ring $R$ is said to have (left) stable range 1 (written $\operatorname{sr}(a)=$ 1) if $R a+R b=R$ (for any $b \in R$ ) implies that $a+r b$ is a unit for some $r \in R$. If $r$ can, in fact, be chosen to be an idempotent, we say that $a$ has (left) idempotent stable range 1 , and write isr $(a)=1$. If $\operatorname{sr}(a)=1$ (resp. isr $(a)=1)$ for all $a \in R$, we'll write $\operatorname{sr}(R)=1$ (resp. $\operatorname{isr}(R)=1$ ). The notion of $\operatorname{isr}(R)=1$ is due to Huanyin Chen [3], who proved its left-right symmetry. Much earlier, the left-right symmetry of $\operatorname{sr}(R)=1$ was proved by Vaserstein in [11].

The notion of clean elements is closely related to the notion of idempotent stable range 1 , since taking $b=-1$ in the above definitions shows that isr $(a)=1$ implies that $a$ is clean. In Theorem 3.3, we'll prove that, if $\operatorname{sr}(R)=1$, then any regular element $a \in R$ has isr $(a)=1$; in particular, $a$ is clean. This implies that all unitregular rings have idempotent stable range 1, and leads to a refinement (Theorem 3.6) of the Camillo-Khurana characterization of unit-regular rings in [2] in terms of the cleanness property. As a by-product of the above results, we show (in Theorem 3.7) that a semilocal ring $R$ is semiperfect iff isr $(R)=1$.

In Section 3, Theorem 3.3 is derived from Theorem 3.1, which formulates our main results in a convenient module-theoretic setting. The proof of the latter theorem rests on the construction of an idempotent endomorphism of a module $M$ using a criterion (Proposition 2.2) for the existence of a common direct complement for two given direct summands of $M$. Here, Warfield's results (in [12]) on modules with the substitution property are not only needed, but also used to their fullest potential.

Throughout this paper, $R$ denotes an arbitrary ring with unity. We'll write $\mathrm{U}(R)$ for the group of units of $R$, and $\operatorname{reg}(R)$ for the set of (von Neumann) regular elements of $R$. Following [7], a module $M$ is said to have IC (the "internal cancellation" property) if $M=C \oplus D=C^{\prime} \oplus D^{\prime}$ and $C \cong C^{\prime}$ imply that $D \cong D^{\prime}$. A ring $R$ is said to be IC if the right module $R_{R}$ is IC. This notion is left-right symmetric according to [7]. For other unexplained terms in ring theory and a general survey on some of the background material pertinent to this paper, see [8] and [9].

\section{Preparatory Results}

In this section, we lay the ground work for the proofs of the main results in Section 3.

Proposition 2.1 Let $R$ be any ring, with $R a+R b=R$, where $a \in \operatorname{reg}(R)$. If $x \in R$ is any element such that axa $=a$, then $b(1-x a) \in \operatorname{reg}(R)$.

Proof Let $e$ be the idempotent $x a$, and let $f=1-e$, for which $a f=0$. Right multiplying $R a+R b=R$ by $f$ gives $R b f=R f$. Since this is a direct summand of ${ }_{R} R, b f \in \operatorname{reg}(R)$. For a more mundane (but "constructive") proof, fix an equation $r a+s b=1$. Left and right multiplying this by $f$ gives $f(s b) f=f$. Thus, $(b f) s(b f)=b f$, showing again that $b f \in \operatorname{reg}(R)$.

The next proposition gives a criterion for two submodules of a given module to have a common direct complement. This result can be gleaned from [1, Prop. 5.5], 
although the statement in [1] is not quite couched in the way we want. To make this part of our paper self-contained, we restate this result in a more convenient form, and also give it a conceptually simpler proof.

Proposition 2.2 Let $M_{k}$ be a right module over any ring $k$, and let $R=\operatorname{End}_{k}(M)$. For any submodules $C, C^{\prime} \subseteq M$, the following are equivalent:

(1) $C, C^{\prime}$ have a common direct complement in $M$.

(2) There exists an idempotent $e \in R$ such that $\operatorname{im}(e)=C$, and $e: C^{\prime} \rightarrow C$ is an isomorphism.

Proof

(1) $\Rightarrow$ (2) Take a submodule $D$ such that $M=C \oplus D=C^{\prime} \oplus D$, and let $e \in R$ be the projection of $M$ onto $C$ with kernel $D$. Then $e: C^{\prime} \rightarrow C$ is injective since $C^{\prime} \cap \operatorname{ker}(e)=0$, and surjective since $C=e(M)=$ $e\left(C^{\prime}+D\right)=e\left(C^{\prime}\right)$.

(2) $\Rightarrow$ (1) Suppose $e \in R$ exists as in (2). Let $D:=\operatorname{ker}(e)$, so $M=\operatorname{im}(e) \oplus$ $\operatorname{ker}(e)=C \oplus D$. We finish by proving that $M=C^{\prime} \oplus D$. Since $e: C^{\prime} \rightarrow$ $C$ is injective, we have already $0=C^{\prime} \cap \operatorname{ker}(e)=C^{\prime} \cap D$. Finally, $e(C)=C=e\left(C^{\prime}\right) \Rightarrow C \subseteq C^{\prime}+D$. Thus, $C^{\prime}+D \supseteq C+D=M$, as desired.

Corollary 2.3 Let $R=\operatorname{End}_{k}(M)$ as in (2.2), and let $C, C^{\prime}$ be direct summands of $M$, with $C \cong C^{\prime}$ and $\operatorname{sr}\left(\operatorname{End}_{k}(C)\right)=1$. Then there exists an idempotent $e \in R$ such that $\operatorname{im}(e)=C$, and $e: C^{\prime} \rightarrow C$ is an isomorphism.

Proof Given that $\operatorname{sr}\left(\operatorname{End}_{k}(C)\right)=1$, the module $C$ has the substitution property in the sense of Warfield [12]. Therefore, by Warfield's result [12, Thm. 2.1], $C$ and $C^{\prime}$ have a common direct complement in $M$. Applying Proposition 2.2, we get the idempotent $e \in R$ with the two required properties.

\section{Proofs of the Main Results}

We come now to the first main result in this paper.

Theorem 3.1 Let $R=\operatorname{End}_{k}(M)$ where $M$ is a right $k$-module with IC (internal cancellation property) over a ring $k$. Let $R a+R b=R$, where $a \in \operatorname{reg}(R)$ and $b \in$ $R$. If $\operatorname{sr}\left(\operatorname{End}_{k}(\operatorname{ker}(a))\right)=1$ or $\operatorname{sr}\left(\operatorname{End}_{k}(\operatorname{im}(a))\right)=1$, then there exists an idempotent $e \in R$ such that (1) $a+e b \in \mathrm{U}(R)$, and (2) $M=\operatorname{im}(a) \oplus \operatorname{im}(e)$.

Proof Say $a=a x a$, where $x \in R$. This implies that $K:=\operatorname{ker}(a)$ and $I:=\operatorname{im}(a)$ are direct summands of $M$, so $M=K \oplus D=C \oplus I$ for suitable submodules $C, D \subseteq$ $M$. Since $D \cong I$ (by Noether's isomorphism theorem), the IC property ${ }^{1}$ on $M$

\footnotetext{
${ }^{1}$ The use of the IC property on $M$ here could have been avoided if we assume that $a \in R$ is unitregular, since this is sufficient to give $K \cong C$.
} 
implies that $K \cong C$. On the other hand, $R a+R b=R \Rightarrow K \cap \operatorname{ker}(b)=0$, so the map $b: K \rightarrow b(K)$ is an isomorphism. Now $K=\operatorname{ker}(a)=\operatorname{ker}(x a)=\operatorname{im}(1-x a)$. Therefore,

$$
C^{\prime}:=b(K)=b \cdot \operatorname{im}(1-x a)=b(1-x a) M .
$$

By Proposition 2.1, $b(1-x a) \in \operatorname{reg}(R)$, so $C^{\prime}$ is a direct summand of $M$, with $C^{\prime} \cong$ $K \cong C$. If we assume that $\operatorname{sr}\left(\operatorname{End}_{k}(K)\right)=1$, then $\operatorname{sr}\left(\operatorname{End}_{k}(C)\right)=1$, so Corollary 2.3 gives an idempotent $e \in R$ such that $\operatorname{im}(e)=C$ and $e: C^{\prime} \rightarrow C$ is an isomorphism. If we assume, instead, that $\operatorname{sr}\left(\operatorname{End}_{k}(I)\right)=1$, then writing $M=C^{\prime} \oplus I^{\prime}$ (for some $I^{\prime}$ ) gives $I^{\prime} \cong I$, by the IC property on $M$. Then Warfield's result (loc. cit.) implies that $I$ has the substitution property, and that $C, C^{\prime}$ have a common complement in $M$. Therefore, by Proposition 2.2, the idempotent $e$ exists as before, with $M=$ $I \oplus C=\operatorname{im}(a) \oplus \operatorname{im}(e)$.

With the idempotent $e$ in place (under either assumption), we claim that $u:=a+$ $e b \in \mathrm{U}(R)$. To prove this, we first check that the following diagram is commutative:

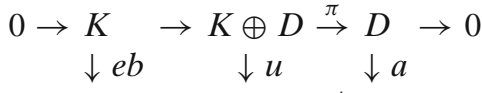

$$
\begin{aligned}
& 0 \rightarrow C \rightarrow C \oplus I \stackrel{\pi^{\prime}}{\rightarrow} I \rightarrow 0
\end{aligned}
$$

The left square is clearly commutative, since $a$ acts as zero on $K$, so $u$ acts as $e b$ on $K$. For the commutativity of the right square, we need to check that $a \pi(m)=\pi^{\prime} u(m)$ for every $m \in M$. If $m \in K$, this is clear as both sides are zero. If $m \in D$, then

$$
\pi^{\prime} u(m)=\pi^{\prime}(a(m)+e b(m))=a(m)=a \pi(m),
$$

since $e b(m) \in \operatorname{im}(e)=C$. Now $e b: K \rightarrow C$ and $a: D \rightarrow I$ are both isomorphisms, so the 5-Lemma implies that $u: M \rightarrow M$ is also an isomorphism, as desired.

Remark 3.2 The statement of Theorem 3.1 can be somewhat simplified if we are willing to use a stronger hypothesis. Instead of assuming that (A) $M$ is IC, and (B) either $\operatorname{sr}\left(\operatorname{End}_{k}(\operatorname{ker}(a))\right)=1$ or $\operatorname{sr}\left(\operatorname{End}_{k}(\operatorname{im}(a))\right)=1$, one may simply assume that $(\mathrm{C}) \operatorname{sr}(R)=1$. Note that $(\mathrm{C}) \Rightarrow(\mathrm{B})$ since $\operatorname{End}_{k}(\operatorname{ker}(a))$ and $\operatorname{End}_{k}(\operatorname{im}(a))$ are corner rings of $R$, and the stable range 1 condition descends to corners, by [11, Thm. 2.8]. A similar argument (using the Evans' Cancellation Theorem [8, (20.11)]) shows that $(\mathrm{C}) \Rightarrow(\mathrm{A})$. In view of these observations, we can now derive the following consequence of Theorem 3.1.

Theorem 3.3 Let $a \in \operatorname{reg}(R)$ where $R$ is a ring with $\operatorname{sr}(R)=1$. Then, whenever $R a+R b=R$, there exists an idempotent $e \in R$ such that $a+e b \in \mathrm{U}(R)$ and $a R \oplus e R=R$. In particular, isr $(a)=1$, and $a$ is clean.

Proof The first conclusion follows from Theorem 3.1 by taking $M$ to be the right module $R_{R}$. Here, End $\left(R_{R}\right)$ is canonically identified with $R$, so Theorem 3.1 applies (thanks to Remark 3.2). Ignoring the extra conclusion $a R \oplus e R=R$, we get isr $(a)=1$. If we apply this property to the equation $R=R a+R(-1)$, we get an idempotent $e \in R$ such that $u:=a+e(-1) \in \mathrm{U}(R)$. Thus, $a=e+u$ is clean. 
Unit-regular rings are known to be of stable range 1 (by a theorem of Kaplansky [5] and Fuchs [4], c. 1971), and clean (by a theorem of Camillo and Khurana [2], c. 2001). Applying Theorem 3.3 to such rings leads to a common improvement on both of these results.

Corollary 3.4 If $R$ is a unit-regular ring, then isr $(R)=1$, and so $R$ is clean.

Remark 3.5 Note that Theorem 3.3 no longer holds if we try to replace the stable range condition $\operatorname{sr}(R)=1$ by the weaker hypothesis that $R$ is an IC ring. A counter example is given by the matrix ring $R=\mathbb{M}_{2}(\mathbb{Z})$. This is an IC ring by [7, (5.9)(1)]. However, in [6], it was shown that unit-regular elements of $R$ need not be clean. Therefore, even for unit-regular elements $a \in R$, although $\operatorname{sr}(a)=1$ (by [7, Thm. 3.5]), we may not have isr $(a)=1$. From the proof of Theorem 3.1 (and Footnote 1), we can ascribe this failure precisely to the fact that the appropriate corner rings associated with the unit-regular element $a$ do not satisfy a stable range one condition.

We complete our discussions on unit-regular rings by restating the CamilloKhurana theorem from [2] ((1) $\Leftrightarrow(2)$ below), and further refining it by adding the equivalent statement (3).

Theorem 3.6 For any ring $R$, the following are equivalent:

(1) $R$ is unit-regular.

(2) For any $a \in R$, there exist $u \in \mathrm{U}(R)$ and an idempotent $e \in R$ such that $a=$ $e+u$ and $a R \cap e R=0$.

(3) Whenever $R a+R b=R$, there exists an idempotent $e \in R$ such that $a+e b \in$ $\mathrm{U}(R)$ and $a R \cap e R=0$.

Proof $(1) \Rightarrow$ (3) follows from Theorem 3.3 (since (1) implies that $R=\operatorname{reg}(R)$ and $\operatorname{sr}(R)=1)$, and (3) $\Rightarrow$ (2) follows by applying (3) with $b=-1$. For (2) $\Rightarrow(1)$, the following proof is only slightly different from the one given in [2]. For $a \in R$ expressed as in (2), we have

$$
a u^{-1} a-a=(e+u) u^{-1} a-a=e u^{-1} a \in e R \cap a R=0 .
$$

This implies that $a=a u^{-1} a$ is unit-regular. (Actually, the above calculation also works if we take $u:=a+e b \in \mathrm{U}(R)$ as in (3).)

Of course, Corollary 3.4 implies that isr $(R)=1$ for every semisimple ring $R$. In the special case $R=\mathbb{M}_{n}(k)$ where $k$ is a division ring, Theorem 3.1 gives an interesting fact in linear algebra when it is applied to the right vector space $M=k^{n}$. Here, $a, b$ are two linear operators on $k^{n}$ with no nonzero common null vector, and Theorem 3.1 gives a "projection" $e$ whose image is complement to that of $a$, such that $a+e b$ is an invertible operator. In particular, $\operatorname{rank}(e)=n-\operatorname{rank}(a)$. Note that the possibility of applying Theorem 3.1 in this manner illustrates the efficacy of the module-theoretic viewpoint used in that theorem.

We conclude this paper with a by-product result on the semiperfect rings of Bass $[8, \S 23]$. It is well known (see, e.g. [10]) that these rings are exactly the exchange rings among the semilocal rings. Using Corollary 3.4 for semisimple rings, we easily arrive 
at the following theorem, which enables us to also recognize the semiperfect rings among the semilocal rings by using a suitable type of stable range 1 condition.

Theorem 3.7 Let $R$ be a semilocal ring. Then $R$ is semiperfect iff isr $(R)=1$.

Proof Let $J=\operatorname{rad}(R)$ be the Jacobson radical of $R$. If $\operatorname{isr}(R)=1$, Chen's result [3, Thm. 9] implies that idempotents can be lifted modulo $J$. Since $R$ is semilocal, this means that $R$ is semiperfect. For the converse, note that $R / J$ is semisimple, so $\operatorname{isr}(R / J)=1$ (as pointed out above). If $R$ is semiperfect, we can lift idempotents modulo $J$. Thus, the same result of Chen cited above gives isr $(R)=1$.

An easy explicit example of a (commutative) semilocal ring $R$ with isr $(R) \neq 1$ is given by the semilocalization of $\mathbb{Z}$ at the two prime ideals $2 \mathbb{Z}$ and $3 \mathbb{Z}$. Here, we have $R a+R b=R$ for $a=4$ and $b=-1$, but the only idempotents $e \in R$ are 1 and 0 , so $a+e b$ can only be 3 or 4 , neither of which is a unit in $R$. (Of course, $R$ is not semiperfect since the idempotents $\overline{3}, \overline{4} \in R / \operatorname{rad}(R)$ fail to lift to idempotents of $R$.)

Acknowledgements This research was carried out during a visit of the first two authors at the University of California, Berkeley. They gratefully acknowledge the hospitality of their host institution. Z. Wang was supported by the Specialized Research Fund for the Doctoral Program of Higher Education for New Teachers (No. 20090092120024). J. Chen was supported by the Specialized Research Fund for the Doctoral Program of Higher Education (No. 200802860024), the NNSF of China (No. 10971024), and the NSF of Jiangsu Province (BK2010393), P.R.C.

Open Access This article is distributed under the terms of the Creative Commons Attribution Noncommercial License which permits any noncommercial use, distribution, and reproduction in any medium, provided the original author(s) and source are credited.

\section{References}

1. Anderson, F., Fuller, K.: Rings and Categories of Modules. Graduate Texts in Math., vol. 13. Springer, Berlin-Heidelberg, New York (1992)

2. Camillo, V., Khurana, D.: A characterization of unit regular rings. Commun. Algebra 29, 22932295 (2001)

3. Chen, H.: Rings with many idempotents. Int. J. Math. 22, 547-558 (1999)

4. Fuchs, L.: On a substitution property of modules. Monatsh. Math. 75, 198-204 (1971)

5. Kaplansky, I.: Bass's first stable range condition. Mimeographed notes (1971)

6. Khurana, D., Lam, T.Y.: Clean matrices and unit-regular matrices. J. Algebra 280, 683-698 (2004)

7. Khurana, D., Lam, T.Y.: Rings with internal cancellation (with an Appendix by R.G. Swan). J. Algebra 284, 203-235 (2005)

8. Lam, T.Y.: A first course in noncommutative rings, 2nd edn. In: Graduate Texts in Math., vol. 131. Springer, Berlin-Heidelberg, New York (2001)

9. Lam, T.Y.: A crash course on stable range, cancellation, substitution, and exchange. J. Algebra and Its Appl. 3, 301-343 (2004)

10. Nicholson, W.K.: Lifting idempotents and exchange rings. Trans. Am. Math. Soc. 229, 269-278 (1977)

11. Vaserstein, L.N.: Bass's first stable range condition. J. Pure Appl. Algebra 34, 319-330 (1984)

12. Warfield, R.B.: Cancellation of modules and groups and stable range of endomorphism rings. Pac. J. Math. 91, 457-485 (1980) 